J Am Chem Soc. 2016 May 18; 138(19): 6119-6122. doi:10.1021/jacs.6b02479.

\title{
The Significance of Multivalent Bonding Motifs and "Bond Order” in DNA-Directed Nanoparticle Crystallization
}

\author{
Ryan V. Thaner ${ }^{\dagger, \S}$, Ibrahim Eryazici ${ }^{\dagger, \S}$, Robert J. Macfarlane ${ }^{\dagger}$, Keith A. Brown $^{\dagger}$, Byeongdu \\ Lee ${ }^{\ddagger}$, SonBinh T. Nguyen ${ }^{*}, \dagger$, and Chad A. Mirkin ${ }^{*} \dagger$ \\ tDepartment of Chemistry and International Institute for Nanotechnology, Northwestern \\ University, 2145 Sheridan Road, Evanston, Illinois 60208, United States \\ $\ddagger$ X-ray Science Division, Advanced Photon Source, Argonne National Laboratory, Argonne, Illinois \\ 60439, United States
}

\begin{abstract}
Multivalent oligonucleotide-based bonding elements have been synthesized and studied for the assembly and crystallization of gold nanoparticles. Through the use of organic branching points, divalent and trivalent DNA linkers were readily incorporated into the oligonucleotide shells that define DNA-NPs and compared to monovalent systems. These multivalent bonding motifs enable the change of "bond strength" between particles and therefore modulate the effective "bond order." In addition, the improved accessibility of strands between neighboring particles, either due to multivalency or modifications to increase strand flexibility, gives rise to superlattices with less strain in the crystallites compared to traditional designs. Furthermore, the increased availability and number of binding modes also provide a new variable that allows previously unobserved crystal structures to be synthesized, as evidenced by the formation of a thorium phosphide superlattice.
\end{abstract}

\begin{abstract}
Almost two decades ago, we reported that polyvalent, DNA-functionalized gold nanoparticles (DNA-NPs) can be programmably assembled via sequence-specific DNA interactions to yield macroscopic aggregates and more recently highly crystalline structures. These materials exhibit plasmonic properties and cooperative behavior that are ideal for a variety of uses in molecular diagnostics. By replacing the nanoparticle core with a rigid organic molecule, we also have systematically studied the cooperative binding characteristics of molecularly pure multivalent nucleic acid constructs. While both of these types of DNA conjugates have illustrated the significance of multivalency in cooperative behavior, the effect is highly amplified in DNA-NP assemblies where hundreds of DNA interactions can occur between NPs. Remarkably, depending upon particle-particle contact
\end{abstract}

\footnotetext{
*Corresponding Authors: chadnano@northwestern.edu; stn@northwestern.edu.

$\$$ Author Contributions

These authors contributed equally.

Supporting Information

The Supporting Information is available free of charge on the ACS Publications website at DOI: 10.1021/jacs.6b02479.

Experimental details and data (PDF)

Notes

The authors declare no competing financial interest.
} 
area, even a single base pair (bp) can stabilize particle assemblies due to the effects of polyvalency. Indeed, the combination of a polyvalent scaffold and a weak individual interparticle interaction strength allows DNA-NPs of arbitrary core composition to behave as "programmable atom equivalents" (PAEs) in the synthesis of hundreds of different crystalline lattices with unique combinations of spacing and symmetry.' Notably, both the NP "atom" and oligonucleotide "bond" can be independently manipulated such that building block identity and bonding behavior can be decoupled. This highly modular platform has prompted us to explore the possibility of creating multiple-bond analogs by incorporating branched organic molecules into the DNA-based bonding elements with the potential for discovering new properties and making new structures (Figure 1A).

Herein, we report the synthesis of NP-based PAEs with branched binding motifs capable of forming DNA "multiple bonds" and their use in the formation of low-strain superlattices. By incorporating branched organic molecules into the traditional linker design, a diversity of bonding modalities can be accessed and provides an additional level of polyvalency at the periphery of PAEs. These complex binding motifs manifest in the formation of higher quality crystals than those obtained via conventional linear designs as well as the previously unobserved thorium phosphide $\left(\mathrm{Th}_{3} \mathrm{P}_{4}, I \overline{4} 3 d\right)$ lattice symmetry.

By design, PAEs consist of three components: a NP core, a dense surface layer of thiolmodified DNA, and a "shell" of DNA and a "shell" of DNA linkers. In a typical assembly experiment, these DNA-NPs were combined with a DNA linker, which comprises a recognition sequence complementary to the particle-bound strand, an unpaired "flexor" region, and a short, single-stranded region termed the "sticky end" that engages in interparticle hybridization events. In our "multiple-bond" design, branching points were incorporated into the DNA linker after the recognition sequence such that each linker possesses two (doubler) or three (trebler) sticky ends (Figure 1A). This design gives rise to a form of valency derived from the DNA itself as opposed to using NP shape and dense oligonucleotide packing to direct the DNA bonding elements. It also should be noted that the multivalent linkers studied herein were typically synthesized with a " $\mathrm{T}_{3}$ " flexor region consisting of three unpaired thymine bases, as opposed to the traditional single-base design, as this has been shown to be optimal in directing DNA interactions in analogous branched organic-DNA hybrid molecular assemblies. We also investigated variations in this flexor region ranging from a standard unpaired A-base to up to 18 ethylene glycol chains (see Supporting Information (SI), Tables S4-S7), which have previously been shown to improve the extent of long-range order or relative crystal quality. Both of these modifications to the traditional linker design should increase the accessibility of sticky ends at the periphery of PAEs, which in turn would provide optimal environments to maximize polyvalent interactions, resulting in improved crystal quality.

As a starting point, commercially available AuNPs with diameters of 10 and $15 \mathrm{~nm}$ were densely functionalized with $5^{\prime}$-thiol modified ssDNA consisting of a [PEG6 $]_{2}$ nonbonding region and a 18-bp sequence using a previously established salt-aging method (see SI, Sections S1-S5 for complete description of all methods, sequences, and strand characterization). A predetermined amount of the appropriate type of linker was then added to each type of DNA-coated NP. Upon combining the complementary particle types (i.e., 
trebler with trebler), hybridization events between the sticky ends induced precipitation of the particles into a disordered aggregate. It is important to note that while multivalent linkers are capable of having the sticky ends bind in the ideal fashion as depicted in Figure 1A, there are other potential binding modes (e.g., hybridization between one or two sticky ends of a trebler but not necessarily all three), and it can be assumed that all of these potential interactions collectively contribute to the observed behavior presented herein.

The use of the doubler and trebler linkers greatly improved the thermal stability of the assynthesized DNA-NP assemblies and dramatically sharpened the melting profiles compared to the traditional systems. When the melting temperatures $\left(T_{\mathrm{m}}\right)$ are plotted for all three linker types, a clear trend is observed for each set of NP diameters: the $T_{\mathrm{m}}$ increases with the number of sticky ends per linker (Figure 1B). These data suggest that multivalent sticky end designs can lead to appreciable increases in thermal stability that are analogous to the increase in bond strengths between two partner atoms in a molecule as the bond order increases. (Detailed data and analysis for both $T_{\mathrm{m}}$ and transition breadth can be found in the SI, including the effects of different flexor types: Tables S3-S7, Figures S45-S46. See also the discussion that follows Figure S45). We note that von Kiedrowski and others have shown that multiple hybridization events in DNA-based molecular systems can greatly stabilize the resulting assembled structures and drawn a similar multiple-bond analogy. For the purposes of brevity and simplicity, the remainder of this manuscript will focus on the contrast between the linear and trebler systems where the differences in all observed behavior were the most prominent.

The notable differences in melting behavior upon the introduction of either branching or flexible units into DNA linkers should have a significant impact on the NP crystallization behavior under annealing conditions. In addition, we hypothesized that having an "accommodating," high density of sticky ends in the potential interaction volumes of neighboring PAEs would facilitate crystal formation and potentially improve crystal quality. Two-component superlattices composed of 10 and $15 \mathrm{~nm}$ AuNPs were prepared by annealing the aggregates around their characteristic $T_{\mathrm{m}}$ values, as determined in the abovementioned experiments, yielding body-centered cubic (bcc) superlattices when the AuNPs were the same or cesium chloridetype $(\mathrm{CsCl})$ symmetries when the components are of different sizes. Confirmation of these lattice symmetries as well as other structural information was obtained using synchrotron-based small-angle X-ray scattering (SAXS). Differences in the extent of long-range order are manifested in the sharpness and number of the scattering peaks, where sharper and well-resolved peaks indicate a higher quality crystal (i.e., those with less strain and where the NPs are not significantly deviated from the ideal positions in the lattices.

The scattering pattern for the $\mathrm{CsCl}$ structure formed from the trebler system is visibly higher quality than that for the linear linkers (Figure 2A; see Figure S48 for doubler SAXS data), in accordance with our hypothesis. Such differences can be quantified using a Williamson-Hall (W-H) analysis, which enables the two main sources of peak broadening, finite grain size, and microstrain, to be deconvoluted through line-shape analysis (see SI page S27 for details and Figure S49 for example of analysis). Remarkably, the superlattices composed of treblerT3 linkers have $5 \times$ less strain ( strain $=0.7 \pm 0.4 \times 10^{-3}$ ) than the analogous linear-A systems 
(strain $=5.1 \pm 0.6 \times 10^{-3}$ ), suggesting that the multivalent linkers enable PAEs to situate themselves closer to their ideal lattice positions than linear systems. For each of the three lattice symmetries ( 15 and $10 \mathrm{~nm} \mathrm{bcc} ; 15-10 \mathrm{~nm} \mathrm{CsCl}$ ) that we examined, a plot of the average strain as a function of the type of linker employed (Figure 2B, a total 95 analyses) clearly illustrates the lower quality and broad variation of crystals assembled with the traditional linear-A design, where the amount of strain and deviations from sample-tosample are the most varied compared to its more flexible counterparts. Notably, each data point represents the average of a variety of linker loadings for each system, further illustrating how the incorporation of flexible moieties imparts an "accommodating" environment for heterogeneities across systems.

While the trebler branching unit provides a means to generate high-quality crystals in a consistent manner, previous work has demonstrated that the incorporation of flexible linkers into the PAE design through other chemical modifications also improves crystal quality (see additional discussion in the SI, section S2). When considering the effect of flexibility on crystallization using strictly linear linkers, we also observe that a PEG flexor imparts less strain into the system while simultaneously minimizing the deviations among various samples. This similarity suggests that the incorporation of flexibility into the linker design, in other forms, provides enough "tolerance" to the system to facilitate the particles arranging themselves closer to their ideal positions as well as accommodates sources of polydispersity (e.g., NP size variations, differences in linker loading) without compromising superlattice quality. However, when considering the temperature at which each sample was annealed relative to its $T_{\mathrm{m}}$, there are clear differences between these two sources of linker flexibility. Specifically, linear systems must be annealed right at or in some cases above their $T_{\mathrm{m}}$ 's in order to achieve high-quality crystals, while trebler systems can be annealed several degrees below their $T_{\mathrm{m}}$ values without any noticeable reduction in crystal quality (Figure 2C).' Such a difference is an important practical consideration since aggregate melting typically occurs over a small temperature range and can completely disrupt the structural integrity of the superlattice.

Finally, while attempting to assemble a $\mathrm{CsCl}$ superlattice with 10 and $15 \mathrm{~nm}$ AuNP cores (but comparable hydrodynamic radii) using trebler linkers, we observed a new crystalline phase that corresponds to the $1 \overline{4} 3 d$ space group (Figure 3; the cubic unit cell is isostructural with $\mathrm{Th}_{3} \mathrm{P}_{4}$ ). This unexpected and complex lattice structure was initially observed in atomic systems when lithium was exposed to high-pressure conditions; to our knowledge, this lattice has never been observed in DNA-directed crystallization and only once previously in a colloidal system. This new $\mathrm{Th}_{3} \mathrm{P}_{4}$-type structure was consistently observed (over $20 \times$ with slight variations in DNA design) and only when using treblers. Compared to the other binary superlattices previously formed using DNA, this structure is significantly more complex with a large unit cell (lattice parameter of $93.59 \mathrm{~nm}$ ) containing $1215 \mathrm{~nm}$ and $1610 \mathrm{~nm}$ particles as opposed to the lattice parameter of $38.24 \mathrm{~nm}$ and one-to-one particle ratio for the analogous $\mathrm{CsCl}$ unit cell.

Interestingly, the inorganic volume fraction of PAEs in the new $\mathrm{Th}_{3} \mathrm{P}_{4}$-type lattice is lower than that for $\mathrm{CsCl}$ (0.036 vs 0.041). However, when the volume fraction of only the $15 \mathrm{~nm}$ $\mathrm{PAE}$ is considered, the new structure is more dense than $\mathrm{CsCl}(0.035$ vs 0.032$)$. While the 
previously mentioned melting transitions indicate that replacing linear linkers with branched units increases attractive interactions between individual DNA strands due to multivalent binding, this new phase with lower packing density suggests that it also increases repulsive interactions between PAEs as a whole. This is further suggested by the fact that the centerto-center distance between the 10 and $15 \mathrm{~nm}$ AuNPs in the new phase is smaller $(32.39 \mathrm{~nm}$, compared with the $33.11 \mathrm{~nm}$ distance observed in the $\mathrm{CsCl}$ structure), meaning the DNA is $3.5 \%$ shorter in length. An in situ crystallization experiment was carried out to determine the relative stabilities of these two lattices and the nature of their phase boundary (see Figure S53). When monitoring the scattering pattern of the initial disordered aggregate as it transitions to a crystalline arrangement upon annealing, it was observed that both the new phase and $\mathrm{CsCl}$ initially formed simultaneously. However, over time, the peaks corresponding to $\mathrm{CsCl}$ disappeared and only the scattering pattern of the new phase remained, suggesting that it is the thermodynamically favored structure. Such a result is counter to what is typically observed in these systems where the densest arrangement of PAEs is favored (see SI, Figure S52A,B for SAXS data of systems with various loadings and flexor types), but is again consistent with the hypothesis of increased repulsive interactions between PAEs.

In conclusion, the use of organic branching moieties in the linkers that define PAEs allows for the realization of new building blocks that provide a type of controllable "bond order" in this field. Such structures also allow one to modulate the DNA-based valency in NP assembly, which presents a means to control particle interactions beyond geometric parameters such as particle size or hydrodynamic radius and has significant consequences on the observed crystallization path. Indeed, such building blocks have now led to the discovery of the $\mathrm{Th}_{3} \mathrm{P}_{4}$ lattice symmetry, one not previously observed in the programmable assembly field. The realization of this new structure is both exciting and challenging as it moves beyond the predictable realm established by previous design rules. This result points to the need to develop additional computational and experimental tools to accurately model and predict the formation of such complex architectures and, in turn, understand how multivalency changes the crystallization pathway. Moving forward, this new design paradigm will have important implications as research efforts shift to the use of PAEs with low linker density or minimal sites for DNA attachment as well as multitiered structures that necessitate gradients of interaction strengths using the same sequence-specific, sticky end interactions.

\section{Supplementary Material}

Refer to Web version on PubMed Central for supplementary material.

\section{Acknowledgments}

This material is based upon work supported by the following awards: Air Force Office of Scientific Research FA9550-11-1-0275 and FA9550-12-1-0280; the Center for Cancer Nano-technology Excellence initiative of the National Institutes of Health U54 CA151880. R.V.T. gratefully acknowledges an NSF Graduate Research Fellowship. This research used resources of the Advanced Photon Source, a U.S. Department of Energy (DOE) Office of Science User Facility operated for the DOE Office of Science by Argonne National Laboratory under contract no. DE-AC02-06CH11357. 


\section{References}

1. (a) Mirkin CA, Letsinger RL, Mucic RC, Storhoff JJ. Nature. 1996; 382:607. [PubMed: 8757129] (b) Jones MR, Seeman NC, Mirkin CA. Science. 2015; 347:1260901. [PubMed: 25700524]

2. (a) Cutler JI, Zhang K, Zheng D, Auyeung E, Prigodich AE, Mirkin CA. J Am Chem Soc. 2011; 133:9254. [PubMed: 21630678] (b) Howes PD, Chandrawati R, Stevens MM. Science. 2014; 346:1247390. [PubMed: 25278614] (c) Rosi NL, Mirkin CA. Chem Rev. 2005; 105:1547. [PubMed: 15826019] (d) Anker JN, Hall WP, Lyandres O, Shah NC, Zhao J, Van Duyne RP. Nat Mater. 2008; 7:442. [PubMed: 18497851]

3. (a) Eryazici I, Prytkova TR, Schatz GC, Nguyen ST. J Am Chem Soc. 2010; 132:17068. [PubMed: 21073153] (b) Prytkova TR, Eryazici I, Stepp BR, Nguyen ST, Schatz GC. J Phys Chem B. 2010; 114:2627. [PubMed: 20136069] (c) Long H, Kudlay A, Schatz GC. J Phys Chem B. 2006; 110:2918. [PubMed: 16471902] (d) Kudlay A, Gibbs JM, Schatz GC, Nguyen ST, Olvera de la Cruz M. J Phys Chem B. 2007; 111:1610. [PubMed: 17256893] (e) Aldaye FA, Sleiman HF. J Am Chem Soc. 2007; 129:13376. [PubMed: 17939666] (f) McLaughlin CK, Hamblin GD, Sleiman HF. Chem Soc Rev. 2011; 40:5647. [PubMed: 22012315] (g) McLaughlin CK, Hamblin GD, Aldaye FA, Yang H, Sleiman HF. Chem Commun. 2011; 47:8925.(h) Meng M, Ahlborn C, Bauer M, Plietzsch O, Soomro SA, Singh A, Muller T, Wenzel W, Brase S, Richert C. ChemBioChem. 2009; 10:1335. [PubMed: 19422011] (i) Greschner AA, Toader V, Sleiman HF. J Am Chem Soc. 2012; 134:14382. [PubMed: 22873572]

4. (a) Jin R, Wu G, Li Z, Mirkin CA, Schatz GC. J Am Chem Soc. 2003; 125:1643. [PubMed: 12568626] (b) Macfarlane RJ, Thaner RV, Brown KA, Zhang J, Lee B, Nguyen ST, Mirkin CA. Proc Natl Acad Sci U S A. 2014; 111:14995. [PubMed: 25298535] (c) Hurst SJ, Hill HD, Mirkin CA. J Am Chem Soc. 2008; 130:12192. [PubMed: 18710229]

5. (a) Macfarlane RJ, O’Brien MN, Petrosko SH, Mirkin CA. Angew Chem Int Ed. 2013; 52:5688.(b) Macfarlane RJ, Lee B, Jones MR, Harris N, Schatz GC, Mirkin CA. Science. 2011; 334:204. [PubMed: 21998382] (c) Macfarlane RJ, Jones MR, Lee B, Auyeung E, Mirkin CA. Science. 2013; 341:1222. [PubMed: 23970559] (d) Nykypanchuk D, Maye MM, van der Lelie D, Gang O. Nature. 2008; 451:549. [PubMed: 18235496] (e) Liu W, Tagawa M, Xin HL, Wang T, Emamy H, Li H, Yager KG, Starr FW, Tkachenko AV, Gang O. Science. 2016; 351:582. [PubMed: 26912698] (f) Brodin JD, Auyeung E, Mirkin CA. Proc Natl Acad Sci U S A. 2015; 112:4564. [PubMed: 25831510]

6. Shchepinov MS, Udalova IA, Bridgman AJ, Southern EM. Nucleic Acids Res. 1997; 25:4447. [PubMed: 9358151]

7. (a) O'Brien MN, Jones MR, Lee B, Mirkin CA. Nat Mater. 2015; 14:833. [PubMed: 26006002] (b) Jones MR, Macfarlane RJ, Lee B, Zhang J, Young KL, Senesi AJ, Mirkin CA. Nat Mater. 2010; 9:913. [PubMed: 20890281] (c) Lu F, Yager KG, Zhang Y, Xin H, Gang O. Nat Commun. 2015; 6:6912. [PubMed: 25903309]

8. Eryazici I, Yildirim I, Schatz GC, Nguyen ST. J Am Chem Soc. 2012; 134:7450. [PubMed: 22428783]

9. (a) Park SY, Lytton-Jean AKR, Lee B, Weigand S, Schatz GC, Mirkin CA. Nature. 2008; 451:553. [PubMed: 18235497] (b) Senesi AJ, Eichelsdoerfer DJ, Brown KA, Lee B, Auyeung E, Choi CHJ, Macfarlane RJ, Young KL, Mirkin CA. Adv Mater. 2014; 26:7235. [PubMed: 25244608]

10. Hurst SJ, Lytton-Jean AKR, Mirkin CA. Anal Chem. 2006; 78:8313. [PubMed: 17165821]

11. (a) Diaz JA, Gibbs-Davis JM. Small. 2013; 9:2862. [PubMed: 23341260] (b) Díaz JA, Grewer DM, Gibbs-Davis JM. Small. 2012; 8:873. [PubMed: 22228478]

12. (a) Scheffler M, Dorenbeck A, Jordan S, Wustefeld M, von Kiedrowski G. Angew Chem Int Ed. 1999; 38:3311.(b) Shchepinov MS, Mir KU, Elder JK, Frank-Kamenetskii MD, Southern EM. Nucleic Acids Res. 1999; 27:3035. [PubMed: 10454597] (c) Hong BJ, Cho VY, Bleher R, Schatz GC, Nguyen ST. J Am Chem Soc. 2015; 137:13381. [PubMed: 26398097]

13. (a) Scardi P, Leoni M, Delhez R. J Appl Crystallogr. 2004; 37:381.(b) Williamson GK, Hall WH. Acta Metall. 1953; 1:22.

14. (a) Hanfland M, Syassen K, Christensen NE, Novikov DL. Nature. 2000; 408:174. [PubMed: 11089965] (b) Henzie J, Grunwald M, Widmer-Cooper A, Geissler PL, Yang PD. Nat Mater. 2012; 11:131. [PubMed: 22101811] 
(A)

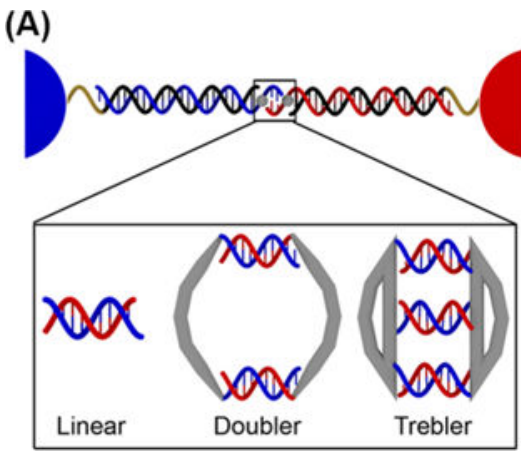

(B)

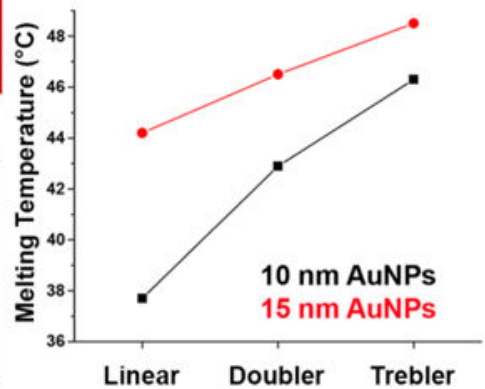

Figure 1.

(A) A schematic representation of NP-based PAEs with tunable bond strengths via branching linkers: the gold NP core (blue and red spheres), a dense surface layer of hexanethiol-modified DNA comprising a [PEG6 $]_{2}$ nonbinding region (gold segment), and an 18-bp sequence (black segment) as well as a DNA linker (red and blue segments). The linker comprises an 18-bp complementary sequence attached to a branching point (gray sphere), a flexor region, and single-stranded "sticky ends" (3'-TTTCCTT and 3'-AAGGAAA) that drive NP assembly via DNA hybridization. (B) Increasing melting temperatures were observed for two sets of NPs with different diameters as the "bond order" or number of sticky ends per linker was increased. For each AuNP size, concentrations were identical, and linker loadings were found to be similar (see SI for DNA loading densities). 

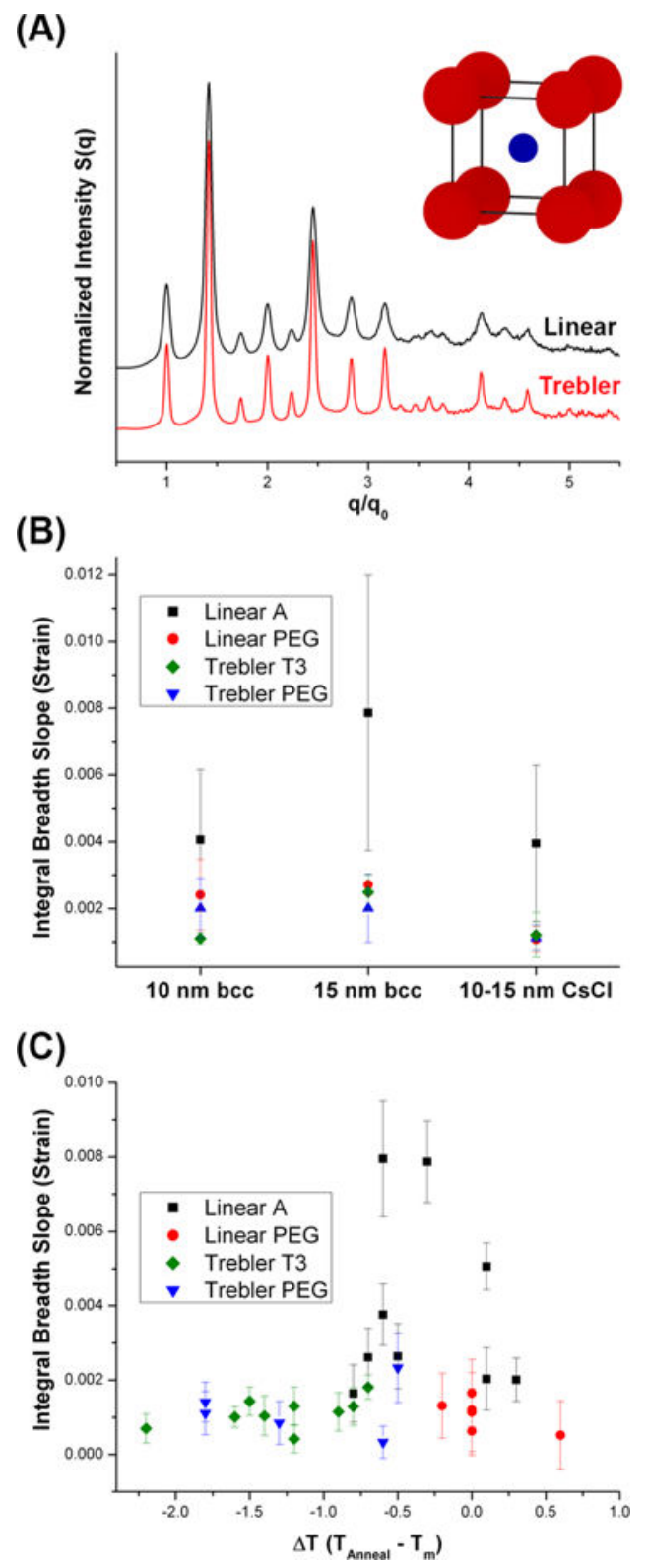

Figure 2.

(A) Comparison of SAXS patterns of CsCl-type superlattices (inset = a depiction of unit cell) formed from PAEs with 10 and $15 \mathrm{~nm}$ diameter cores. The traditional linear-A system is of lower quality than that assembled with the trebler-T3 linkers. (B) Plot of average amounts of microstrain for each lattice symmetry as a function of linker design where each data point encompasses all linker loadings for each system. The incorporation of flexibility enables consistent formation of higher quality lattices relative to those with the linear-A linker design. (C) Plot of strain values for all $\mathrm{CsCl}$ systems based on the temperature at which each system was annealed relative to its $T_{\mathrm{m}}$ (i.e., the difference between the temperature determined by optical melting analysis and that used to anneal the system). (In such a plot, systems that appear to have different strains at the same $\Delta T$ value are unique assemblies that were annealed at different absolute temperatures.) Trebler linkers allow for 
the formation of high-quality crystals when annealed several degrees below the $T_{\mathrm{m}}$. In contrast, systems containing linear linkers must be annealed right at or just above their respective $T_{\mathrm{m}}$ values for the crystals to form. (See SI, Figures S47 and S50 for the analogous bcc plots and pages S27-S28 for discussion of the propagation of the error bars during the W-H analysis.). 


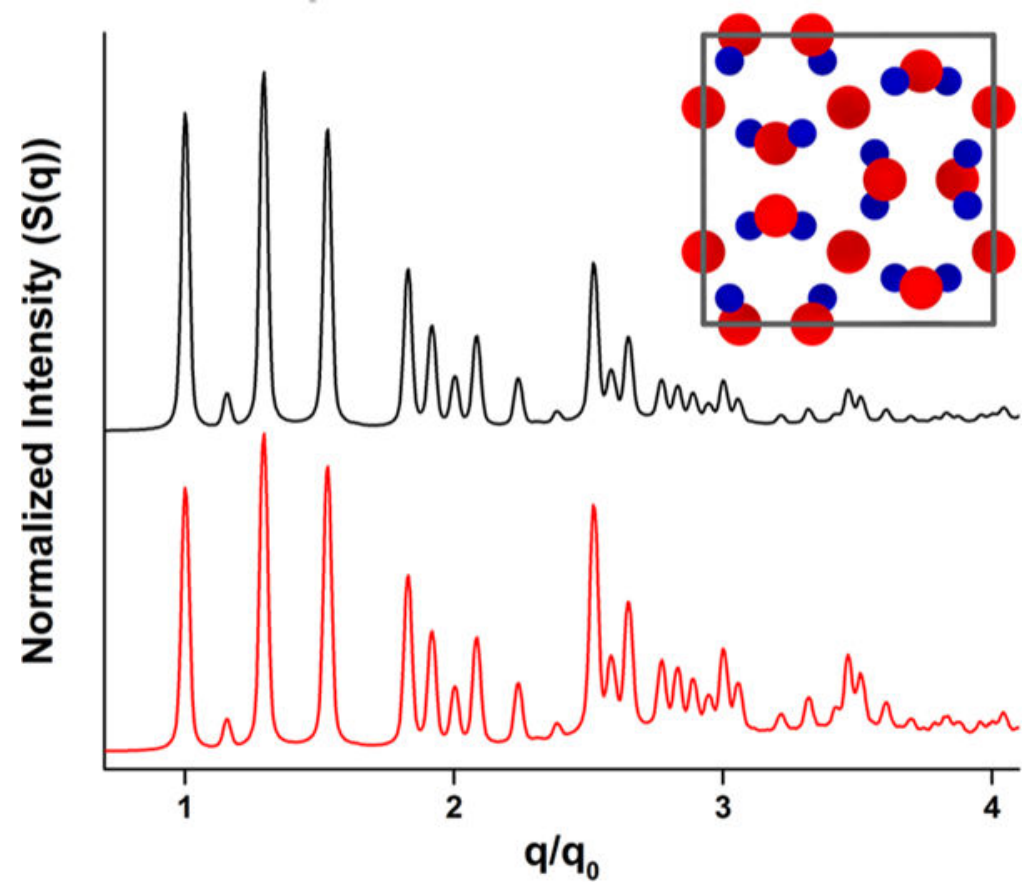

Figure 3.

SAXS data for the $\mathrm{Th}_{3} \mathrm{P}_{4}$ structure observed when using trebler linkers (red, theoretical; black, observed). The unit cell is depicted in the inset with a perspective down the $z$-axis (blue spheres $=10 \mathrm{~nm}$ AuNPs, Wyckoff position of 16c; red spheres $=15 \mathrm{~nm}$ AuNPs, Wyckoff positon of $12 \mathrm{a} ; \mathrm{x}=0.087$ ). One $15 \mathrm{~nm}$ particle is enclosed in a triangle-based distorted dodecahedron composed of eight $10 \mathrm{~nm}$ particles (Figure S51). 